Article

\title{
Evaluation of Legionella pneumophila Decrease in Hot Water Network of Four Hospital Buildings after Installation of Electron Time Flow Taps
}

\author{
Michele Totaro, Tommaso Mariotti, Costanza Bisordi, Erica De Vita, Paola Valentini, \\ Anna Laura Costa, Beatrice Casini ${ }^{D}$, Gaetano Privitera and Angelo Baggiani * \\ Department of Translational Research and New Technologies in Medicine and Surgery, University of Pisa, \\ 56126 Pisa PI, Italy; micheleto@hotmail.it (M.T.); tommaso.mariotti@med.unipi.it (T.M.); \\ costanza.bisordi@med.unipi.it (C.B.); erica.devita@med.unipi.it (E.D.V.); paola.valentini@dps.unipi.it (P.V.); \\ anna.costa@med.unipi.it (A.L.C.); beatrice.casini@med.unipi.it (B.C.); gaetano.privitera@med.unipi.it (G.P.) \\ * Correspondence: angelo.baggiani@med.unipi.it; Tel.: +39-050-2213583; Fax: +39-050-2213588
}

Received: 26 November 2019; Accepted: 9 January 2020; Published: 11 January 2020

\begin{abstract}
Legionella spp. control is a critical issue in hospital with old water networks. Chemical disinfection methods are applied as a control measure over prolonged time periods, but Legionella may be resistant to chemical agents in pipeworks with low flow and frequent water stagnation. We evaluated Legionella spp. colonization in the hot water network of Italian hospitals after the installation of time flow taps (TFTs). In the period between 2017 and 2019, TFTs were installed in four hospital water networks. They were programmed in order to obtain a hot water flow of 192 L/day from each TFTs. A continuous chlorination system (chlorine dioxide) and a cold water pre-filtration device were applied in all the buildings. Before and after TFT installation, Legionella spp. was investigated at scheduled times. Before TFT installation, Legionella pneumophila was detected in all the hospitals with counts ranging from $2 \times 10^{2}$ to $1.4 \times 10^{5} \mathrm{CFU} / \mathrm{L}$. After TFT installation, a loss in Legionella pneumophila culturability was always achieved in the period between $24 \mathrm{~h}$ and 15 days. Total chlorine concentration $\left(\mathrm{Cl}_{2}\right)$ was detected in the range between 0.23 and $0.36 \mathrm{mg} / \mathrm{L}$ while temperature values were from 44.8 to $53.2^{\circ} \mathrm{C}$. TFTs together with chemical disinfection represent a method which improve water quality and disinfectant efficacy, reducing Legionella colonization in dead-end sections.
\end{abstract}

Keywords: Legionella; electron time flow taps; hospital water network; chlorine dioxide

\section{Introduction}

Legionella bacteria are environmental organisms found in water, soil, and other environments. Typically, they are transmitted to humans via the inhalation of contaminated aerosols and they may cause fulminant pneumonia or mild Pontiac fever. In particular, nosocomial Legionella spp. infection may affect immunosuppressed, transplant patients, and people undergoing aggressive chemotherapy [1,2].

According to the European Center for Disease Prevention and Control (ECDC), the rate of Legionnaires' disease in Europe in 2017 was 18 cases per million inhabitants. During the same period, in Europe, 9 out of the 30 countries reported 28 legionellosis outbreaks, either community or hospital acquired. A 30\% increase in the number of reported cases in Europe was observed in 2017 compared with 2016, with an increase in the overall European notification rate from 1.4 to 1.8 per 100,000 population $[3,4]$. 
The latest Italian data, related to 2017, give 2014 cases of Legionnaires' disease in the Italian population, corresponding to a notification rate of 33.2 cases per million inhabitants. It must be noted that 2017 has seen an increase of $26 \%$ in the cases when compared with the previous three years. Of the 2014 notified cases, $124(6.2 \%)$ were hospital acquired while 1580 (78.5\%) were community-acquired infections [5].

International and Italian guidelines for Legionnaires' disease control suggest that large hospital water networks and high-volume hot water storage tanks provide optimal conditions for Legionella colonization; the complexity of the water distribution systems and the presence of old and corroded pipelines and dead-end branches may promote the growth of Legionella in hospital water networks [6,7]. These critical points in water distribution systems cause a low water flow with a higher likelihood of biofilm development. Once microorganisms enter a system, they may be incorporated into biofilm, which is a primary cause of scarce water quality. Biofilm provides a nutrient source and protection from unfavorable environmental conditions. Several variables impact biofilm formation, including fluctuating water usage and transient stagnation [8-11]. In a previous study, we reported the experience of an Italian hospital where a cost-effectiveness strategy for Legionella control in the hot water distribution system was applied with continuous disinfection treatment and the installation of time flow taps (TFTs) in correspondence of all the dead-end branches, with the aim to increase the water flow of 196 L/day. Legionella pneumophila sg2-14 was initially detected in all the sampling points despite the presence of chemical disinfection. After eight months of TFTs activity, Legionella spp. was not detected anymore [12].

Based on that preliminary experience, the aim of this study is the application of the same method in teaching hospitals hosting high-risk patients. In this manuscript, we report the data related to the decrease in Legionella spp. contamination in the hot water networks of four hospitals located in Northwestern Tuscany (Italy) from 2017 to 2019.

\section{Materials and Methods}

\subsection{Settings}

The healthcare setting, called Hospital 1, is a 60-bed Internal and Emergency Medicine Hospital. This structure has been active since 1950. The hospital architectural structure is a monoblock with a central plate on four levels. Hospital 2 (Oncology Unit) is a monoblock with a central plate with three levels which has been active since 1972. It has 30 beds. Hospital 3 is a 320-bed General and Transplant Surgery Unit which has been active since 2007. It is organized in five blocks with four levels. Hospital 4 is a 11-bed Senology Unit which has been active since 1987. It has a central plate on four levels.

\subsection{Water Disinfection}

Within the Water Safety Plan (WSP) implementation program, hot water system treatment and a systematic monitoring program with sampling at points of use began in all hospitals.

The hospital WSP is a self-control manual aimed to manage the microbiological water risk from waterborne pathogens, such as Legionella spp. This protocol is a technical guide which describes the control critical points of the water systems (dead-end branches, pipework materials, devices, etc.), the water samplings for microbiological tests, the disinfection procedure of water networks, and all responsibilities related to plant management.

Municipal drinking water was pre-filtered before entering the hospital hot water distribution system. Despite the old and galvanized steel-made pipelines, the hot water network was treated with continuous chlorine dioxide disinfection.

Continuous disinfection with chlorine dioxide was applied in water networks. This method was used one day before TFT installation.

In hospital water stations, a chemical feed pump, chlorine solution tank, and chlorine residual monitoring kit were installed. 
Hot water was recirculated in order to obtain an energy saving. After the identification of all the dead-end branches present in the hot water distribution system, TFTs were installed corresponding to the most critical points.

They present an adjustable flowmeter in order to measure the scheduled water flow. TFTs were programmed in order to obtain a hot water flushing of 1 minute every $2 \mathrm{~h}$ (192 liters per day from each TFT), as described elsewhere [12].

The TFTs, continuous chlorine dioxide disinfection, and municipal water pre-filtration plant were simultaneously installed in Hospital 1, Hospital 2, and Hospital 4. Four TFTs were placed in these buildings.

In Hospital 3, the continuous chlorination method was already present before TFT installation. Seven TFTs were simultaneously installed.

\subsection{Hot Water Samplings and Legionella spp. Detection}

According to all the hospital WSP programs, the hot water distribution system was sampled at the recirculation point of the central heating system $(\mathrm{R})$, at the exit of the hot water production boiler (B), at each TFT, and at two points of use without TFTs (P1 and P2).

TFTs were installed close to some dead-end branches of the water networks (storage rooms, kitchens, and lockers rooms), while P1 and P2 were chosen from medical stays or surgery areas.

Legionella spp. detection and physical-chemical parameter assessment (total chlorine concentration, $\mathrm{pH}$, and water temperature) were performed according to the following schedule:

- $\quad$ One week before TFT installation (Time 0 or T0);

- The day after TFT installation (Time 1 or T1);

- $\quad$ Seven days after TFT installation (Time 2 or T2);

- $\quad$ Fifteen days after TFT installation (Time 3 or T3);

- One month after TFT installation, and then regularly on a monthly basis (Time 4 or T4, Time 5 or T5, etc.).

All physical-chemical parameter were measured in triplicate before and after TFT installation.

Samplings were performed after five minutes of water flushing. Hot water was collected in sterile bottles having sodium thiosulfate $(20 \mathrm{mg} / \mathrm{L})$. Temperature and total chlorine values were detected after the flushing.

Total chlorine concentration was determined by the colorimetric Visocolor $\mathrm{HE}^{\circledR}$ test (Macherey-Nagel, Düren, Germany).

Isolation of Legionella spp. in hot water samples was performed as suggested by the international standards procedure ISO11731 [13]. Briefly, one liter of water was filtrated through a $0.2 \mu \mathrm{m}$ diameter membrane (Millipore, Billerica, MA, USA); the membrane was then immersed in $10 \mathrm{~mL}$ of the same water and sonicated for $5 \mathrm{~min}$, allowing the detachment of the cells. The suspension was thermally inactivated by incubation at $50{ }^{\circ} \mathrm{C}$ for $30 \mathrm{~min}$. Afterwards, $0.1 \mathrm{~mL}$ of the suspension was plated on Legionella BMPA selective medium (Oxoid Ltd., Basingstoke, Hampshire, UK) and the plates were placed in one incubator at $37^{\circ} \mathrm{C}$ for $7-10$ days within jars with a modified atmosphere $\left(2.5 \% \mathrm{CO}_{2}\right)$ Finally, $10 \%$ of the Legionella-suspected colonies that grew on the medium were subjected to confirmation test using BCYE medium and BCYE without cysteine medium (Oxoid Ltd., Basingstoke, Hampshire, UK). Confirmed colonies were subjected to species and serogroup identification analysis using a multipurpose latex agglutination test (Legionella Latex Test, Oxoid Ltd., Basingstoke, Hampshire, UK).

Chemical samplings were taken at the same points chosen for microbiology tests. Chemical analyses were performed one week before TFT installation, six months after TFT installation, and then regularly on a six-monthly basis. Chemical parameters such as iron ions, zinc ions, and trihalomethanes (THMs) were determined as established by Council Directive 98/83/EC [14]. 


\subsection{Statistical Analysis}

The Kolmogorov-Smirnov test was performed to verify normality of distributions. For each hospital, Kruskal-Wallis test and Dunn's test were used to compare Legionella spp. counts detected before and after TFT installations, as described elsewhere [12]. Power tests were carried out to estimate the sample sizes. The 1-beta values of the significant variables were $>0.8$, demonstrating that sample sizes were acceptable. The statistical analysis was fulfilled using IBM SPSS software package version 17.0.1.

\section{Results}

\subsection{Legionella spp.}

In Hospital 1, before the TFT installation, high Legionella pneumophila sg2-16 concentrations were detected in all the tested water point of use. Concentrations ranging from $2 \times 10^{2}$ to $2.6 \times 10^{4} \mathrm{CFU} / \mathrm{L}$, with a mean value of $9.2 \times 10^{3} \pm 1.1 \times 10^{3} \mathrm{CFU} / \mathrm{L}$, were observed in the hot water samples. Following the implementation of TFTs and water treatment, from September 2017 onwards, we detected Legionella pneumophila sg2-16 for at least seven days, with concentrations ranging from $1 \times 10^{2}$ to $8.8 \times 10^{4} \mathrm{CFU} / \mathrm{L}$ and a mean count of $3.2 \times 10^{4} \pm 2.9 \times 10^{4} \mathrm{CFU} / \mathrm{L}$. A significant decrease in Legionella pneumophila sg2-16 contamination was obtained at the fifteenth and the thirtieth day (T3 and T4). After two months, Legionella spp. was no longer recovered (Figure 1).

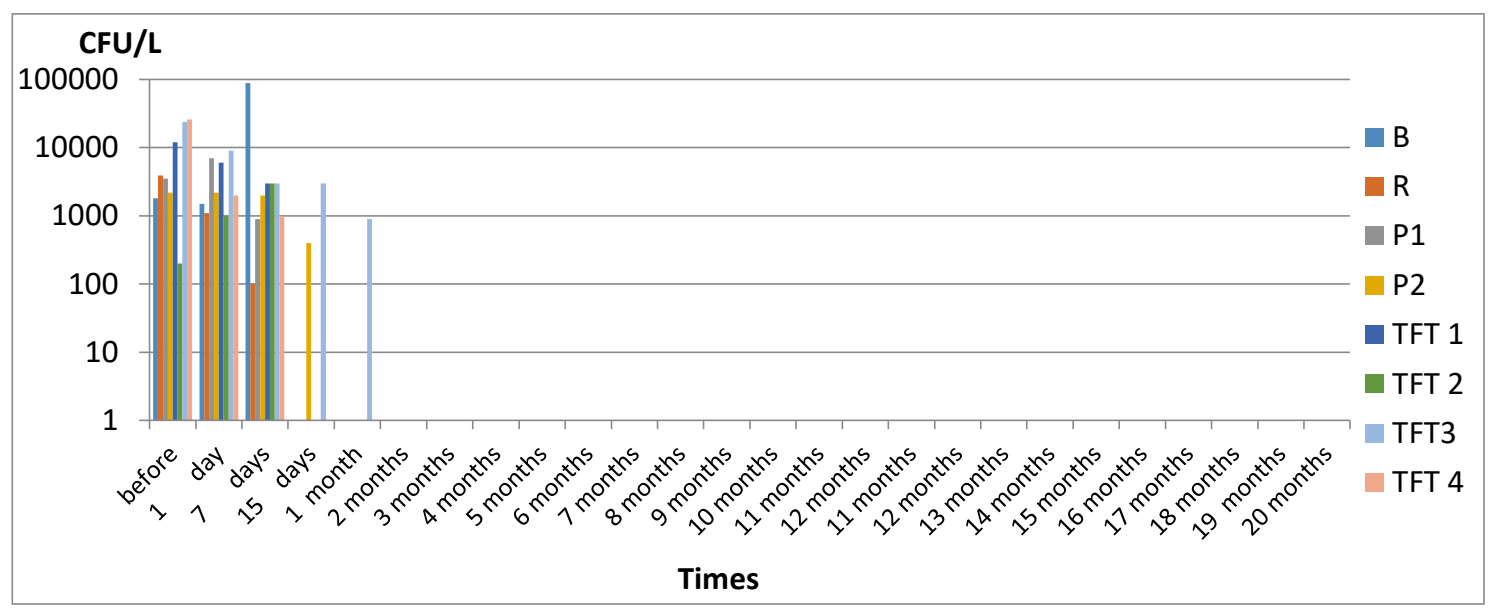

Figure 1. Legionella pneumophila sg2-16 counts at all the sampling points during the whole period of the study in Hospital 1. $\mathrm{B}=$ boiler; $\mathrm{R}=$ recirculation; $\mathrm{P} 1=$ point $1 ; \mathrm{P} 2$ = point 2 ; TFT1 = time flow tap 1; TFT2 = time flow tap 2; TFT3 = time flow tap 3; TFT4 = time flow tap 4.

High Legionella pneumophila sg1 concentrations were detected in Hospital 2 before TFT installation (from $3 \times 10^{2}$ to $1.5 \times 10^{3} \mathrm{CFU} / \mathrm{L}$, mean of $8.8 \times 10^{2} \pm 1.5 \times 10^{2} \mathrm{CFU} / \mathrm{L}$ ). From April 2018, Legionella pneumophila sg1 was detected for at least four days, with a mean concentration of $1.8 \times 10^{2} \mathrm{CFU} / \mathrm{L}$. Legionella spp. could not be detected after seven days (T2), (Figure 2).

In Hospital 3, despite the presence of the continuous chemical chlorination, Legionella pneumophila sg1 was detected in all the hot water samples at T0. Legionella concentrations ranged from $2.1 \times 10^{4}$ to $1.4 \times 10^{5} \mathrm{CFU} / \mathrm{L}$ (mean of $5.5 \times 10^{4} \pm 3.1 \times 10^{3} \mathrm{CFU} / \mathrm{L}$ ). After TFT installation, from November 2018, Legionella pneumophila sg1 was still present for at least two weeks (from $1 \times 10^{2}$ to $2.4 \times 10^{3} \mathrm{CFU} / \mathrm{L}$ ). Absence of Legionella pneumophila sg1 was obtained from the fifteenth day (T3) (Figure 3). 


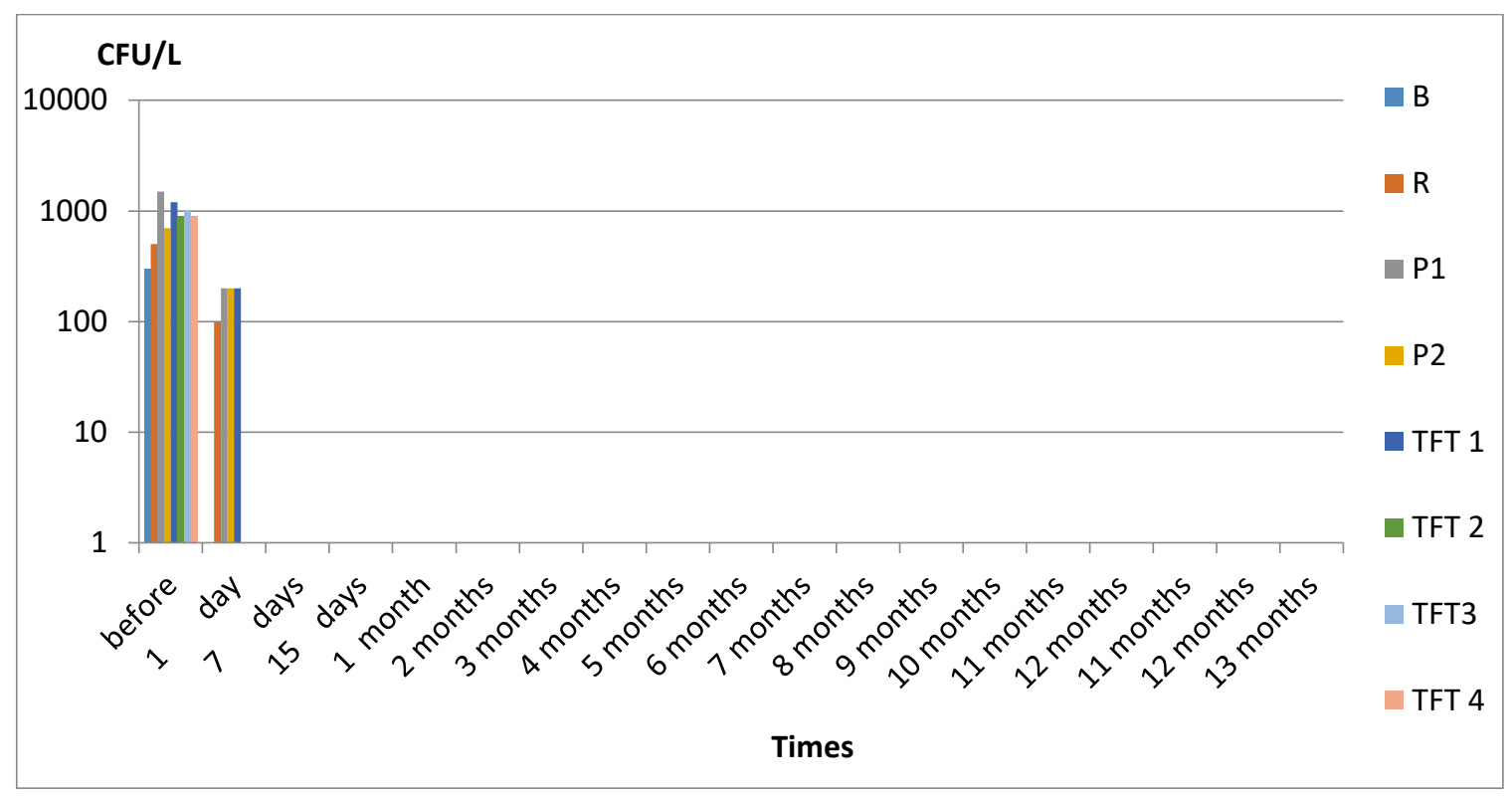

Figure 2. Legionella pneumophila sg1 counts at all the sampling points during the whole period of the study in Hospital 2. $(\mathrm{B}=$ boiler; $\mathrm{R}=$ recirculation; $\mathrm{P} 1=$ point $1 ; \mathrm{P} 2=$ point 2 ; $\mathrm{TFT} 1=$ time flow tap 1 ; TFT2 = time flow tap 2; TFT3 = time flow tap 3; TFT4 = time flow tap 4).

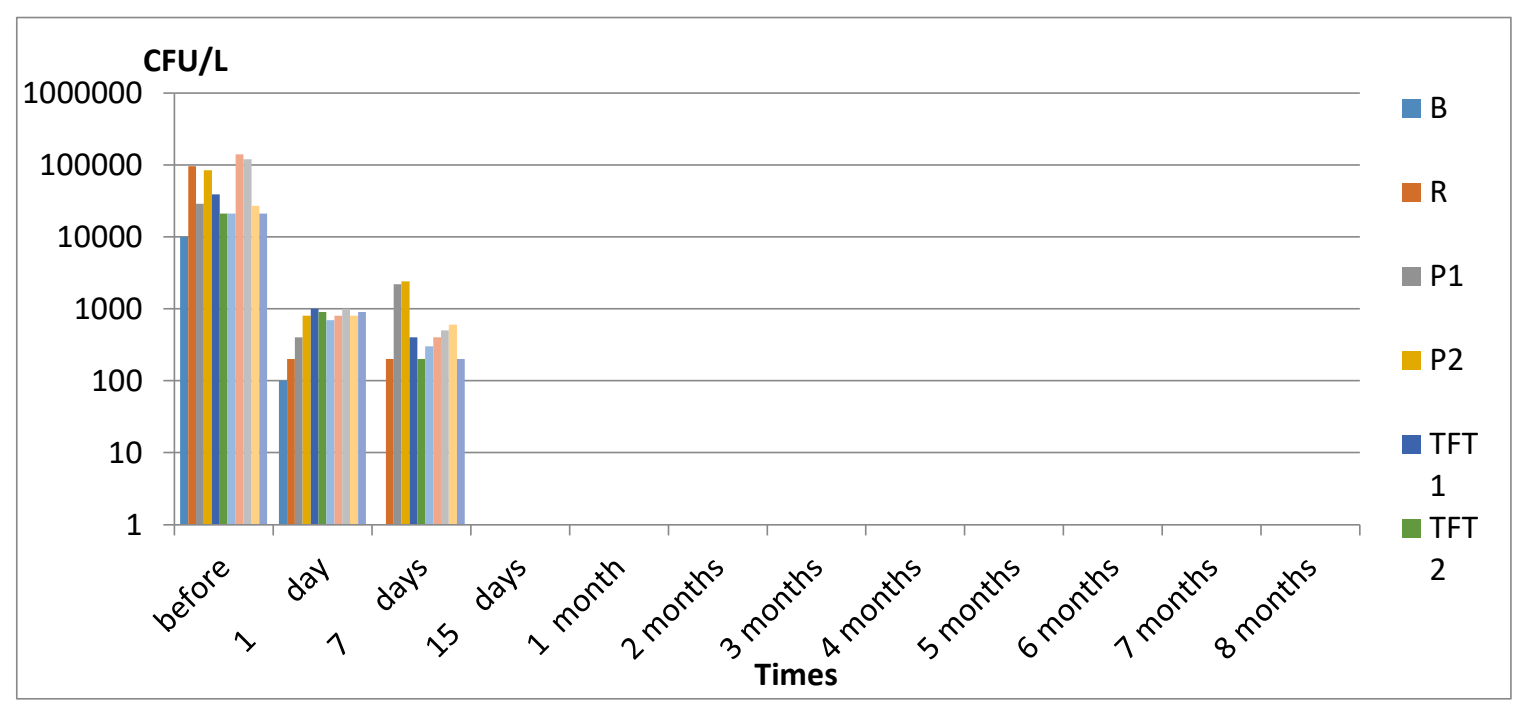

Figure 3. Legionella pneumophila sg1 counts at all the sampling points during the whole period of the study in Hospital $3 . \mathrm{B}=$ boiler; $\mathrm{R}=$ recirculation; $\mathrm{P} 1=$ point $1 ; \mathrm{P} 2=$ point 2 ; $\mathrm{TFT} 1=$ time flow tap 1 ; TFT2 = time flow tap 2; TFT3 = time flow tap 3; TFT4 = time flow tap 4; TFT5 = time flow tap 5; TFT6 = time flow tap 6; TFT7 = time flow tap 7. 
Before the TFT installation, Legionella pneumophila sg1 was detected in Hospital 4. Legionella counts ranged from $1 \times 10^{2}$ to $1.9 \times 10^{4} \mathrm{CFU} / \mathrm{L}$, with a mean value of $9.9 \times 10^{3} \pm 2.8 \times 10^{3} \mathrm{CFU} / \mathrm{L}$. Following the implementation of TFTs and water treatment, from February 2018, Legionella pneumophila sg1 was detected for one day $\left(2.2 \times 10^{2} \mathrm{CFU} / \mathrm{L}\right)$. Legionella pneumophila sg1 was no longer detected from the seventh day (T2) (Figure 4).

In all hospitals, statistically significant reductions of Legionella counts were detected from the period were TFTs were absent to the period when the water flushing was increased $(p<0.001)$.

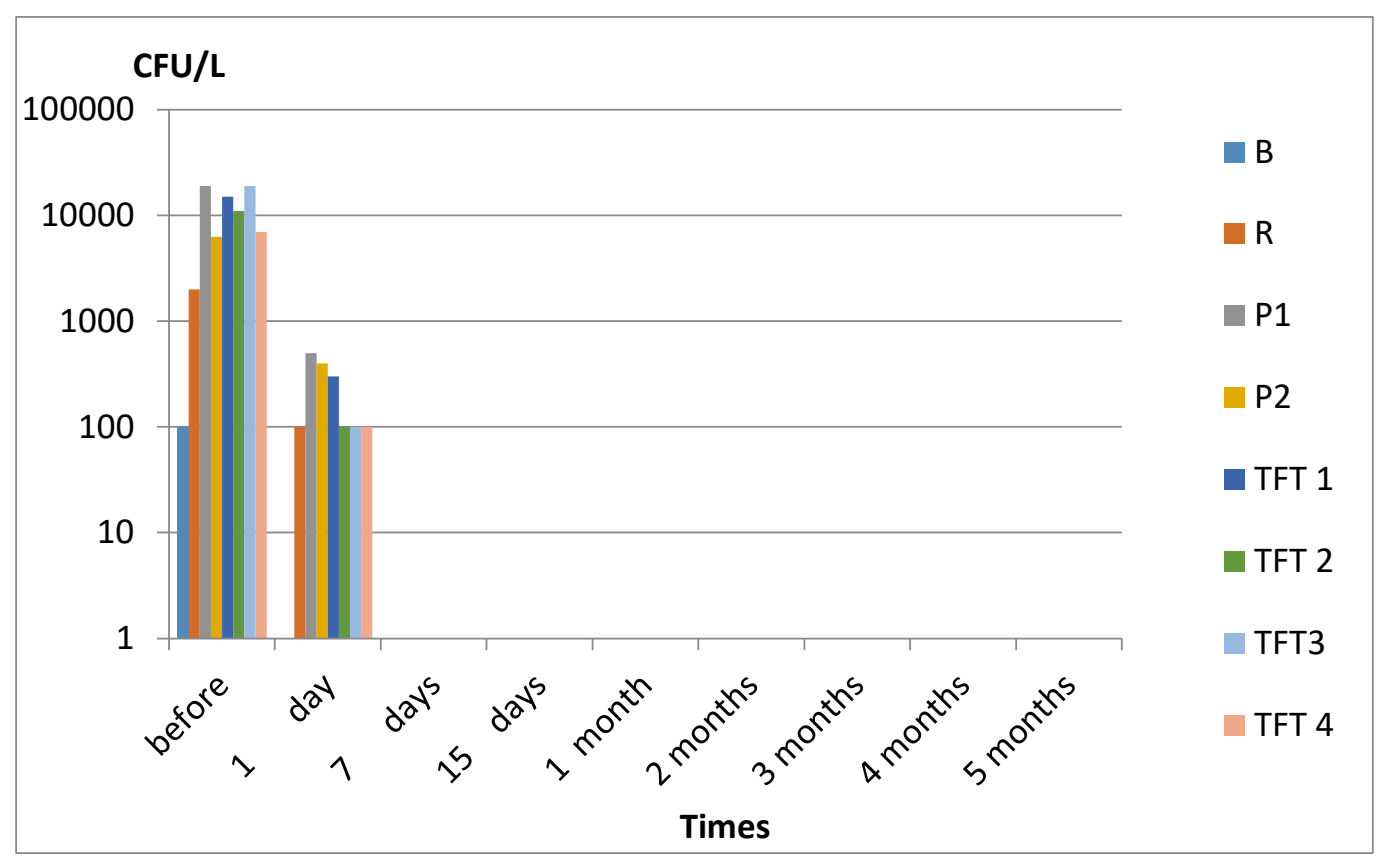

Figure 4. Legionella pneumophila sg1 counts at all the sampling points during the whole period of the study in Hospital 4 . $\mathrm{B}=$ boiler; $\mathrm{R}=$ recirculation; $\mathrm{P} 1=$ point $1 ; \mathrm{P} 2=$ point 2 ; $\mathrm{TFT} 1=$ time flow tap 1; TFT2 = time flow tap 2; TFT3 = time flow tap 3; TFT4 = time flow tap 4.

\subsection{Physical-Chemical Parameters}

In all hospitals, good chemical quality was observed in all hot water samples, and all values were within the limits recommended by Council Directive 98/83/EC [14].

In Hospital 1, the hot water temperature ranged from $36.4 \pm 1.9$ to $48.4 \pm 2.8^{\circ} \mathrm{C}$ before TFT installation. From the first day, mean temperature values ranged from $45.5 \pm 3.2$ and $53.2 \pm 1.6^{\circ} \mathrm{C}$ while continuous chlorination was maintained at mean values between $0.23 \pm 0.04$ to $0.31 \pm 0.06 \mathrm{mg} / \mathrm{L}$ (Table 1).

Before TFT installation, the hot water temperature ranged from $35.8 \pm 3.9$ and $43.5 \pm 8.4{ }^{\circ} \mathrm{C}$ in Hospital 2.

After TFT installation, the mean temperature values ranged from $46.6 \pm 2.1$ and $51.4 \pm 1.7^{\circ} \mathrm{C}$, while the continuous chlorination was maintained at mean values between $0.23 \pm 0.06$ to $0.35 \pm 0.04 \mathrm{mg} / \mathrm{L}$ (Table 2). 
Table 1. Mean values of physical-chemical and chemical parameters assessed at each sampling points before and after TFT installation in Hospital 1. $\mathrm{B}=$ boiler; $\mathrm{R}=$ recirculation; $\mathrm{P} 1=$ point 1 ; $\mathrm{P} 2=$ point 2 ; $\mathrm{TFT} 1=$ time flow tap $1 ; \mathrm{TFT} 2=$ time flow tap 2; TFT3 = time flow tap 3; TFT4 = time flow tap 4.

\begin{tabular}{|c|c|c|c|c|c|c|}
\hline \multicolumn{7}{|c|}{ BEFORE TFT INSTALLATION (September 2017) } \\
\hline $\begin{array}{l}\text { SAMPLING } \\
\text { POINT }\end{array}$ & $\begin{array}{l}\text { TOTAL CHLORINE } \\
(\text { Mean } \pm \text { SD mg/L) }\end{array}$ & $\begin{array}{l}\text { TEMPERATURE } \\
\left(\text { Mean } \pm \mathrm{SD}^{\circ} \mathrm{C}\right)\end{array}$ & $\begin{array}{c}\mathrm{pH} \\
(\text { Mean } \pm \mathrm{SD})\end{array}$ & $\begin{array}{c}\text { IRON IONS } \\
(\text { Mean } \pm \text { SD } \mu \mathrm{g} / \mathrm{L})\end{array}$ & $\begin{array}{c}\text { ZINC IONS } \\
(\text { Mean } \pm \text { SD } \mu \mathrm{g} / \mathrm{L})\end{array}$ & $\begin{array}{l}\text { THMs } \\
(\mu \mathrm{g} / \mathrm{L})\end{array}$ \\
\hline B & 0 & $48.4 \pm 2.8$ & $6.52 \pm 0.31$ & $52 \pm 4.5$ & $67 \pm 2.6$ & $<3$ \\
\hline $\mathbf{R}$ & 0 & $38.8 \pm 1.9$ & $6.50 \pm 0.12$ & $69 \pm 4.3$ & $73 \pm 1.9$ & $<3$ \\
\hline P1 & 0 & $38.8 \pm 2.1$ & $6.60 \pm 0.16$ & $57 \pm 3.9$ & $82 \pm 3.8$ & $<3$ \\
\hline P2 & 0 & $36.4 \pm 1.9$ & $6.57 \pm 0.11$ & $98 \pm 2.6$ & $79 \pm 6.2$ & $<3$ \\
\hline TFT 1 & 0 & $39.5 \pm 1.6$ & $6.58 \pm 0.09$ & $74 \pm 5.9$ & $65 \pm 7.1$ & $<3$ \\
\hline TFT 2 & 0 & $37.7 \pm 1.7$ & $6.70 \pm 0.31$ & $61 \pm 6.6$ & $81 \pm 6.0$ & $<3$ \\
\hline TFT 3 & 0 & $38.1 \pm 2.5$ & $6.60 \pm 0.09$ & $69 \pm 5.7$ & $83 \pm 5.7$ & $<3$ \\
\hline TFT 4 & 0 & $37.6 \pm 2.2$ & $6.52 \pm 0.06$ & $70 \pm 4.2$ & $77 \pm 6.4$ & $<3$ \\
\hline \multicolumn{7}{|c|}{ AFTER TFT INSTALLATION (September 2017-July 2019) } \\
\hline $\begin{array}{l}\text { SAMPLING } \\
\text { POINT }\end{array}$ & $\begin{array}{l}\text { TOTAL CHLORINE } \\
(\text { Mean } \pm \text { SD mg/L) }\end{array}$ & $\begin{array}{l}\text { TEMPERATURE } \\
\left(\text { Mean } \pm \text { SD }^{\circ} \mathrm{C}\right)\end{array}$ & $\begin{array}{c}\mathrm{pH} \\
(\text { Mean } \pm \mathrm{SD})\end{array}$ & $\begin{array}{c}\text { IRON IONS } \\
(\text { Mean } \pm \text { SD } \mu \mathrm{g} / \mathrm{L}) \\
\end{array}$ & $\begin{array}{c}\text { ZINC IONS } \\
(\text { Mean } \pm \mathrm{SD} \mu \mathrm{g} / \mathrm{L})\end{array}$ & $\begin{array}{l}\text { THMs } \\
(\mu \mathrm{g} / \mathrm{L})\end{array}$ \\
\hline B & $0.31 \pm 0.06$ & $53.2 \pm 1.6$ & $6.59 \pm 0.18$ & $59 \pm 3.1$ & $74 \pm 3$ & $<3$ \\
\hline $\mathbf{R}$ & $0.23 \pm 0.04$ & $49.6 \pm 2.1$ & $6.60 \pm 0.11$ & $77 \pm 3.6$ & $81 \pm 4.1$ & $<3$ \\
\hline P1 & $0.29 \pm 0.09$ & $47.4 \pm 1.2$ & $6.56 \pm 0.09$ & $63 \pm 2.9$ & $79 \pm 3.3$ & $<3$ \\
\hline P2 & $0.27 \pm 0.09$ & $45.5 \pm 3.2$ & $6.5 \pm 0.08$ & $77 \pm 4.2$ & $66 \pm 4.2$ & $<3$ \\
\hline TFT 1 & $0.26 \pm 0.08$ & $48.6 \pm 2.5$ & $6.58 \pm 0.13$ & $68 \pm 3.8$ & $78 \pm 2.6$ & $<3$ \\
\hline TFT 2 & $0.24 \pm 0.04$ & $49.8 \pm 1.5$ & $6.51 \pm 0.10$ & $71 \pm 4.6$ & $82 \pm 5.2$ & $<3$ \\
\hline TFT 3 & $0.26 \pm 0.05$ & $48.3 \pm 2.1$ & $6.61 \pm 0.07$ & $74 \pm 2.3$ & $84 \pm 3.9$ & $<3$ \\
\hline TFT 4 & $0.26 \pm 0.08$ & $47.3 \pm 2.2$ & $6.58 \pm 0.09$ & $75 \pm 3.3$ & $79 \pm 3.6$ & $<3$ \\
\hline
\end{tabular}

Table 2. Mean values of physical-chemical and chemical parameters assessed at each sampling points before and after TFT installation in Hospital 2. B = boiler; $\mathrm{R}=$ recirculation; $\mathrm{P} 1=$ point $1 ; \mathrm{P} 2$ = point 2; $\mathrm{TFT} 1=$ time flow tap 1; TFT2 = time flow tap 2; TFT3 = time flow tap 3; TFT4 = time flow tap 4.

\begin{tabular}{|c|c|c|c|c|c|c|}
\hline \multicolumn{7}{|c|}{ BEFORE TFT INSTALLATION (April 2018) } \\
\hline $\begin{array}{l}\text { SAMPLING } \\
\text { POINT }\end{array}$ & $\begin{array}{l}\text { TOTAL CHLORINE } \\
(\text { Mean } \pm \text { SD mg/L) }\end{array}$ & $\begin{array}{l}\text { TEMPERATURE } \\
\left(\text { Mean } \pm \mathrm{SD}^{\circ} \mathrm{C}\right)\end{array}$ & $\begin{array}{c}\mathrm{pH} \\
(\text { Mean } \pm \mathrm{SD})\end{array}$ & $\begin{array}{c}\text { IRON IONS } \\
(\text { Mean } \pm \text { SD } \mu \mathrm{g} / \mathrm{L})\end{array}$ & $\begin{array}{c}\text { ZINC IONS } \\
(\text { Mean } \pm \text { SD } \mu \mathrm{g} / \mathrm{L})\end{array}$ & $\begin{array}{l}\text { THMs } \\
(\mu \mathrm{g} / \mathrm{L})\end{array}$ \\
\hline B & 0 & $43.5 \pm 8.4$ & $6.72 \pm 0.18$ & $66 \pm 2.2$ & $64 \pm 1.1$ & $<3$ \\
\hline $\mathbf{R}$ & 0 & $37.6 \pm 9.2$ & $6.70 \pm 0.19$ & $68 \pm 2.1$ & $66 \pm 1.6$ & $<3$ \\
\hline P1 & 0 & $39.2 \pm 5.4$ & $6.74 \pm 0.21$ & $61 \pm 1.9$ & $64 \pm 1.0$ & $<3$ \\
\hline P2 & 0 & $35.8 \pm 3.9$ & $6.80 \pm 0.20$ & $78 \pm 1.1$ & $80 \pm 0.9$ & $<3$ \\
\hline TFT 1 & 0 & $38.8 \pm 4.8$ & $6.78 \pm 0.16$ & $79 \pm 1.8$ & $85 \pm 0.7$ & $<3$ \\
\hline TFT 2 & 0 & $39.4 \pm 5.9$ & $6.78 \pm 0.19$ & $65 \pm 2.0$ & $87 \pm 1.2$ & $<3$ \\
\hline TFT 3 & 0 & $39.1 \pm 4.6$ & $6.76 \pm 0.16$ & $69 \pm 2.2$ & $86 \pm 1.3$ & $<3$ \\
\hline TFT 4 & 0 & $36.9 \pm 6.9$ & $6.80 \pm 0.25$ & $73 \pm 2.1$ & $79 \pm 1.0$ & $<3$ \\
\hline \multicolumn{7}{|c|}{ AFTER TFT INSTALLATION (April 2018-July 2019) } \\
\hline $\begin{array}{l}\text { SAMPLING } \\
\text { POINT }\end{array}$ & $\begin{array}{l}\text { TOTAL CHLORINE } \\
(\text { Mean } \pm \text { SD mg/L) }\end{array}$ & $\begin{array}{l}\text { TEMPERATURE } \\
\left(\text { Mean } \pm \mathrm{SD}^{\circ} \mathrm{C}\right)\end{array}$ & $\begin{array}{c}\mathrm{pH} \\
(\text { Mean } \pm \mathrm{SD})\end{array}$ & $\begin{array}{c}\text { IRON IONS } \\
(\text { Mean } \pm \text { SD } \mu \mathrm{g} / \mathrm{L})\end{array}$ & $\begin{array}{c}\text { ZINC IONS } \\
(\text { Mean } \pm \text { SD } \mu \mathrm{g} / \mathrm{L})\end{array}$ & $\begin{array}{l}\text { THMs } \\
(\mu \mathrm{g} / \mathrm{L})\end{array}$ \\
\hline B & $0.35 \pm 0.07$ & $51.4 \pm 1.7$ & $6.79 \pm 0.11$ & $74 \pm 0.19$ & $63 \pm 0.19$ & $<3$ \\
\hline $\mathbf{R}$ & $0.26 \pm 0.06$ & $47.6 \pm 1.9$ & $6.70 \pm 0.10$ & $69 \pm 0.15$ & $68 \pm 0.13$ & $<3$ \\
\hline P1 & $0.24 \pm 0.09$ & $47.6 \pm 1.4$ & $6.60 \pm 0.10$ & $63 \pm 0.15$ & $66 \pm 0.14$ & $<3$ \\
\hline P2 & $0.23 \pm 0.08$ & $46.6 \pm 2.1$ & $6.62 \pm 0.09$ & $75 \pm 0.11$ & $81 \pm 0.18$ & $<3$ \\
\hline TFT 1 & $0.25 \pm 0.06$ & $47.5 \pm 2.1$ & $6.68 \pm 0.11$ & $79 \pm 0.17$ & $84 \pm 0.17$ & $<3$ \\
\hline TFT 2 & $0.27 \pm 0.05$ & $47.9 \pm 1.6$ & $6.61 \pm 0.14$ & $70 \pm 0.12$ & $82 \pm 0.12$ & $<3$ \\
\hline TFT 3 & $0.26 \pm 0.03$ & $48.4 \pm 1.7$ & $6.64 \pm 0.11$ & $74 \pm 0.17$ & $85 \pm 0.19$ & $<3$ \\
\hline TFT 4 & $0.27 \pm 0.05$ & $46.9 \pm 1.8$ & $6.78 \pm 0.11$ & $76 \pm 0.18$ & $81 \pm 0.11$ & $<3$ \\
\hline
\end{tabular}

In Hospital 3, total chlorine concentrations were between 0 to $0.1 \pm 0.02 \mathrm{mg} / \mathrm{L}$, while hot water temperature values ranged from $32.8 \pm 1.8$ to $50.7 \pm 1.6{ }^{\circ} \mathrm{C}$ before TFT installation. Continuous chlorination was maintained at mean values between $0.25 \pm 0.01$ to $0.40 \pm 0.01 \mathrm{mg} / \mathrm{L}$, while mean temperature values ranged from $44.8 \pm 1.8$ and $50.7 \pm 1.6^{\circ} \mathrm{C}$ (Table 3 ).

In Hospital 4 the water temperature values ranged from $32.4 \pm 1.8$ to $39.9 \pm 3.2^{\circ} \mathrm{C}$ at $\mathrm{T} 0$.

From the first day the mean temperature values ranged from $46.4 \pm 2.2$ to $50.1 \pm 1.9^{\circ} \mathrm{C}$ and the continuous chlorination was maintained at mean values between $0.28 \pm 0.06$ and $0.36 \pm 0.04 \mathrm{mg} / \mathrm{L}$ (Table 4). 
Table 3. Mean values of physical-chemical and chemical parameters assessed at each sampling points before and after TFT installation in Hospital 3. ( $\mathrm{B}=$ boiler; $\mathrm{R}=$ recirculation; $\mathrm{P} 1=$ point $1 ; \mathrm{P} 2=$ point 2 ; TFT1 = time flow tap 1; TFT2 = time flow tap 2; TFT3 = time flow tap 3; TFT4 = time flow tap 4; TFT5 = time flow tap 5; TFT6 = time flow tap 6; TFT7 = time flow tap 7).

\begin{tabular}{|c|c|c|c|c|c|c|}
\hline \multicolumn{7}{|c|}{ BEFORE TFT INSTALLATION (November 2018) } \\
\hline $\begin{array}{l}\text { SAMPLING } \\
\text { POINT }\end{array}$ & $\begin{array}{l}\text { TOTAL CHLORINE } \\
(\text { Mean } \pm \text { SD mg/L) }\end{array}$ & $\begin{array}{l}\text { TEMPERATURE } \\
\left(\text { Mean } \pm \mathrm{SD}^{\circ} \mathrm{C}\right)\end{array}$ & $\begin{array}{c}\mathrm{pH} \\
(\text { Mean } \pm \text { SD) }\end{array}$ & $\begin{array}{c}\text { IRON IONS } \\
(\text { Mean } \pm \text { SD } \mu \mathrm{g} / \mathrm{L})\end{array}$ & $\begin{array}{c}\text { ZINC IONS } \\
(\text { Mean } \pm \text { SD } \mu \mathrm{g} / \mathrm{L})\end{array}$ & $\begin{array}{l}\text { THMs } \\
(\mu \mathrm{g} / \mathrm{L})\end{array}$ \\
\hline B & $0.10 \pm 0.02$ & $40.6 \pm 3.1$ & $6.80 \pm 0.21$ & $55 \pm 0.8$ & $54 \pm 1.6$ & $<3$ \\
\hline $\mathbf{R}$ & $0.05 \pm 0.01$ & $35.8 \pm 2.5$ & $6.78 \pm 0.20$ & $52 \pm 0.9$ & $61 \pm 2.1$ & $<3$ \\
\hline P1 & $0.1 \pm 0.05$ & $40.1 \pm 1.8$ & $6.76 \pm 0.15$ & $51 \pm 1.1$ & $53 \pm 2.2$ & $<3$ \\
\hline $\mathbf{P 2}$ & $0.0 \pm 0.0$ & $42.6 \pm 1.6$ & $6.78 \pm 0.17$ & $60 \pm 0.8$ & $54 \pm 2.5$ & $<3$ \\
\hline TFT 1 & $0.05 \pm 0.01$ & $41.1 \pm 1.5$ & $6.78 \pm 0.17$ & $61 \pm 0.9$ & $61 \pm 2.6$ & $<3$ \\
\hline TFT 2 & $0.05 \pm 0.01$ & $41.9 \pm 2.2$ & $6.75 \pm 0.15$ & $56 \pm 1.2$ & $58 \pm 3.6$ & $<3$ \\
\hline TFT 3 & $0.05 \pm 0.01$ & $42.6 \pm 3.6$ & $6.82 \pm 0.20$ & $55 \pm 1.9$ & $57 \pm 3.4$ & $<3$ \\
\hline TFT 4 & $0.05 \pm 0.01$ & $39.8 \pm 3.2$ & $6.82 \pm 0.19$ & $56 \pm 2.2$ & $55 \pm 5.0$ & $<3$ \\
\hline TFT 5 & $0.05 \pm 0.02$ & $39.5 \pm 3.0$ & $6.79 \pm 0.12$ & $55 \pm 2.6$ & $60 \pm 2.9$ & $<3$ \\
\hline TFT 6 & $0.05 \pm 0.03$ & $39.1 \pm 2.9$ & $6.80 \pm 0.16$ & $54 \pm 2.0$ & $59 \pm 2.3$ & $<3$ \\
\hline TFT 7 & $0.05 \pm 0.01$ & $32.8 \pm 1.8$ & $6.80 \pm 0.09$ & $55 \pm 1.8$ & $56 \pm 3.3$ & $<3$ \\
\hline \multicolumn{7}{|c|}{ AFTER TFT INSTALLATION (November 2018-July 2019) } \\
\hline $\begin{array}{l}\text { SAMPLING } \\
\text { POINT }\end{array}$ & $\begin{array}{l}\text { TOTAL CHLORINE } \\
(\text { Mean } \pm \text { SD mg/L) }\end{array}$ & $\begin{array}{l}\text { TEMPERATURE } \\
\left(\text { Mean } \pm \mathrm{SD}^{\circ} \mathrm{C}\right)\end{array}$ & $\begin{array}{c}\mathrm{pH} \\
(\mathrm{Mean} \pm \mathrm{SD})\end{array}$ & $\begin{array}{c}\text { IRON IONS } \\
(\text { Mean } \pm \text { SD } \mu \mathrm{g} / \mathrm{L})\end{array}$ & $\begin{array}{c}\text { ZINC IONS } \\
(\text { Mean } \pm \text { SD } \mu \mathrm{g} / \mathrm{L})\end{array}$ & $\begin{array}{l}\text { THMs } \\
(\mu \mathrm{g} / \mathrm{L})\end{array}$ \\
\hline B & $0.40 \pm 0.01$ & $50.7 \pm 1.6$ & $6.79 \pm 0.11$ & $59 \pm 0.12$ & $55 \pm 0.12$ & $<3$ \\
\hline $\mathbf{R}$ & $0.25 \pm 0.10$ & $44.8 \pm 1.8$ & $6.70 \pm 0.10$ & $59 \pm 0.16$ & $57 \pm 0.12$ & $<3$ \\
\hline P1 & $0.31 \pm 0.10$ & $46.5 \pm 1.8$ & $6.60 \pm 0.10$ & $61 \pm 0.18$ & $55 \pm 0.11$ & $<3$ \\
\hline $\mathbf{P} 2$ & $0.33 \pm 0.11$ & $49.8 \pm 2.2$ & $6.62 \pm 0.09$ & $65 \pm 0.13$ & $63 \pm 0.19$ & $<3$ \\
\hline TFT 1 & $0.28 \pm 0.12$ & $48.8 \pm 2.0$ & $6.68 \pm 0.11$ & $61 \pm 0.11$ & $62 \pm 0.15$ & $<3$ \\
\hline TFT 2 & $0.30 \pm 0.06$ & $49.1 \pm 1.8$ & $6.61 \pm 0.14$ & $62 \pm 0.10$ & $68 \pm 0.14$ & $<3$ \\
\hline TFT 3 & $0.29 \pm 0.05$ & $48.8 \pm 1.6$ & $6.64 \pm 0.11$ & $63 \pm 0.11$ & $74 \pm 0.10$ & $<3$ \\
\hline TFT 4 & $0.28 \pm 0.03$ & $47.6 \pm 1.6$ & $6.78 \pm 0.11$ & $66 \pm 0.13$ & $61 \pm 0.09$ & $<3$ \\
\hline TFT 5 & $0.32 \pm 0.04$ & $48.6 \pm 1.2$ & $6.80 \pm 0.10$ & $61 \pm 0.09$ & $69 \pm 0.11$ & $<3$ \\
\hline TFT 6 & $0.29 \pm 0.06$ & $47.6 \pm 1.4$ & $6.69 \pm 0.10$ & $59 \pm 0.13$ & $64 \pm 0.11$ & $<3$ \\
\hline TFT 7 & $0.30 \pm 0.02$ & $47.2 \pm 1.3$ & $6.72 \pm 0.11$ & $59 \pm 0.12$ & $66 \pm 0.08$ & $<3$ \\
\hline
\end{tabular}

Table 4. Mean values of physical-chemical and chemical parameters assessed at each sampling points before and after TFT installation in Hospital 4. (B = boiler; $\mathrm{R}=$ recirculation; $\mathrm{P} 1=$ point $1 ; \mathrm{P} 2=$ point 2; $\mathrm{TFT} 1$ = time flow tap 1; TFT2 = time flow tap 2; TFT3 = time flow tap 3; TFT4 = time flow tap 4).

\begin{tabular}{|c|c|c|c|c|c|c|}
\hline \multicolumn{7}{|c|}{ BEFORE TFT INSTALLATION (February 2019) } \\
\hline $\begin{array}{l}\text { SAMPLING } \\
\text { POINT }\end{array}$ & $\begin{array}{l}\text { TOTAL CHLORINE } \\
(\text { Mean } \pm \text { SD mg/L) }\end{array}$ & $\begin{array}{l}\text { TEMPERATURE } \\
\left(\text { Mean } \pm \text { SD }^{\circ} \mathrm{C}\right)\end{array}$ & $\begin{array}{c}\mathrm{pH} \\
(\text { Mean } \pm \mathrm{SD})\end{array}$ & $\begin{array}{c}\text { IRON IONS } \\
(\text { Mean } \pm \text { SD } \mu \mathrm{g} / \mathrm{L})\end{array}$ & $\begin{array}{c}\text { ZINC IONS } \\
(\text { Mean } \pm \text { SD } \mu \mathrm{g} / \mathrm{L})\end{array}$ & $\begin{array}{l}\text { THMs } \\
(\mu \mathrm{g} / \mathrm{L})\end{array}$ \\
\hline B & 0 & $39.9 \pm 3.2$ & $6.81 \pm 0.10$ & $77 \pm 0.50$ & $69 \pm 0.18$ & $<3$ \\
\hline $\mathbf{R}$ & 0 & $37.1 \pm 2.5$ & $6.78 \pm 0.12$ & $81 \pm 0.60$ & $68 \pm 0.20$ & $<3$ \\
\hline P1 & 0 & $35.4 \pm 2.9$ & $6.76 \pm 0.13$ & $76 \pm 0.62$ & $64 \pm 0.16$ & $<3$ \\
\hline P2 & 0 & $34.6 \pm 2.6$ & $6.80 \pm 0.09$ & $74 \pm 0.41$ & $78 \pm 0.17$ & $<3$ \\
\hline TFT 1 & 0 & $33.2 \pm 1.8$ & $6.79 \pm 0.18$ & $75 \pm 0.52$ & $74 \pm 0.24$ & $<3$ \\
\hline TFT 2 & 0 & $32.4 \pm 4.2$ & $6.78 \pm 0.20$ & $71 \pm 0.19$ & $79 \pm 0.20$ & $<3$ \\
\hline TFT 3 & 0 & $33.3 \pm 3.6$ & $6.77 \pm 0.15$ & $79 \pm 0.20$ & $75 \pm 0.19$ & $<3$ \\
\hline TFT 4 & 0 & $34.5 \pm 2.7$ & $6.74 \pm 0.11$ & $81 \pm 0.16$ & $76 \pm 0.11$ & $<3$ \\
\hline \multicolumn{7}{|c|}{ AFTER TFT INSTALLATION (February 2019-July 2019) } \\
\hline $\begin{array}{l}\text { SAMPLING } \\
\text { POINT }\end{array}$ & $\begin{array}{l}\text { TOTAL CHLORINE } \\
(\text { Mean } \pm \text { SD mg/L) }\end{array}$ & $\begin{array}{l}\text { TEMPERATURE } \\
\left(\text { Mean } \pm \mathrm{SD}^{\circ} \mathrm{C}\right)\end{array}$ & $\begin{array}{c}\mathrm{pH} \\
(\text { Mean } \pm \mathrm{SD})\end{array}$ & $\begin{array}{c}\text { IRON IONS } \\
(\text { Mean } \pm \text { SD } \mu \mathrm{g} / \mathrm{L})\end{array}$ & $\begin{array}{c}\text { ZINC IONS } \\
(\text { Mean } \pm \text { SD } \mu \mathrm{g} / \mathrm{L})\end{array}$ & $\begin{array}{l}\text { THMs } \\
(\mu \mathrm{g} / \mathrm{L})\end{array}$ \\
\hline B & $0.36 \pm 0.04$ & $50.1 \pm 1.9$ & $6.83 \pm 0.08$ & $80 \pm 0.14$ & $73 \pm 0.17$ & $<3$ \\
\hline $\mathbf{R}$ & $0.30 \pm 0.04$ & $46.4 \pm 2.2$ & $6.79 \pm 0.08$ & $83 \pm 0.12$ & $71 \pm 0.15$ & $<3$ \\
\hline P1 & $0.34 \pm 0.07$ & $48.5 \pm 1.3$ & $6.79 \pm 0.09$ & $79 \pm 0.09$ & $72 \pm 0.18$ & $<3$ \\
\hline P2 & $0.29 \pm 0.09$ & $49.9 \pm 2.0$ & $6.78 \pm 0.09$ & $76 \pm 0.10$ & $80 \pm 0.19$ & $<3$ \\
\hline TFT 1 & $0.31 \pm 0.07$ & $48.9 \pm 2.1$ & $6.81 \pm 0.06$ & $79 \pm 0.15$ & $80 \pm 0.19$ & $<3$ \\
\hline TFT 2 & $0.32 \pm 0.04$ & $48.2 \pm 1.9$ & $6.70 \pm 0.08$ & $76 \pm 0.14$ & $79 \pm 0.18$ & $<3$ \\
\hline TFT 3 & $0.29 \pm 0.02$ & $47.9 \pm 2.3$ & $6.72 \pm 0.09$ & $83 \pm 0.19$ & $78 \pm 0.16$ & $<3$ \\
\hline TFT 4 & $0.28 \pm 0.06$ & $48.2 \pm 2.2$ & $6.79 \pm 0.08$ & $76 \pm 0.18$ & $77 \pm 0.19$ & $<3$ \\
\hline
\end{tabular}

\section{Discussion}

According to literature, Legionella spp. control is needed in hospital settings, mostly in high-risk areas hosting immunosuppressed patients $[15,16]$. International and Italian guidelines $[6,7]$ recommend 
the application of control measures to ensure good microbiological quality in hospital water networks. The choice of an adequate and continuous physical or chemical disinfection system may prevent the occurrence of waterborne pathogen colonization in hospital water plants [17], but the complete eradication of Legionella spp. seems mostly improbable if the colonization is high and the water network is old, large, and complex, with critical points [18]. In spite of the age of the water plant and the presence of continuous chlorination in Hospital 3, we detected the presence of high Legionella pneumophila concentration in hot water samples. This is due to the low hot water use in the hospital, which caused the persistence of Legionella spp. bacteria in biofilm, as described in our preliminary study [12]. In fact, the presence of dead-end branches in water networks allows the biofilm growth in points where the disinfectant cannot be effective against microorganisms [19].

Despite the different number of hospital levels or beds, the amount of dead-end branches detected in hot water networks were the same, with the exception of Hospital 3. In fact, we installed four TFTs in Hospital 1, Hospital 2, and Hospital 4, while seven TFTs were installed in Hospital 3.

The choice of TFT installation points depends on the kind of subjects (patients, healthcare workers, etc.) present in those areas. We avoided TFT installation in stays areas, surgeries, and medical offices in order to limit the exposition to contaminated aerosol during TFT installation.

These devices were mostly placed in storage rooms, kitchens, and locker rooms.

The TFT installation occurred in correspondence with dead-end sections in all the hospitals and the implementation of continuous chemical chlorination in Hospital 1, Hospital 2, and Hospital 4, and the municipal water pre-filtration devices allowed Legionella pneumophila decontamination during the period between T1 (24 h) and T4 (one month). As shown in Figure 5, Legionella pneumophila colonized all hospitals at T0 and T1. Contaminations persisted in Hospital 1 for one month and in Hospital 3 for seven days, despite the presence of total chlorine concentrations higher than $0.25 \mathrm{mg} / \mathrm{L}$. After two months, Legionella pneumophila was no longer detected. Moreover, in all hospitals, the increase of temperature values was obtained from T0 to T1. This is due to the maintenance works on the hot water networks performed during TFT installation. Considering the low hot water temperatures detected at $\mathrm{T} 0\left(\leq 40^{\circ} \mathrm{C}\right)$, we increased this value, in order to achieve boiler water temperatures of at least of $50{ }^{\circ} \mathrm{C}$. According to recent studies, the increase in hot water temperature, lack of water stagnation, and application of good hydraulic practices play a role in Legionella spp. control in water networks [20-22].

Our results show that water flushing eases the chlorine dioxide efficacy in water networks. A reduction of stagnant water reduces biofilm growth, which is considered as a form of microorganism resistant against chemical disinfectants, such as chlorine dioxide, applied in water networks [23,24].

Literature data has shown the impact of unsteady flow on drinking water quality. Considering that stagnation promotes bacterial accumulation, special attention should be given to the biological quality of drinking water where consumption is variable. Interactions between hydraulics and biofilm structures are complex, and bacteria may be released from the biofilm into water after only $1 \mathrm{~h}$ of stagnation, allowing water quality degradation [22-25].

In conclusion, our study continues the assessment of TFT device applications from our previous work [12]. The aim of the TFTs was to increase the efficacy of the water disinfection system. In fact, TFT installation is considered a good cost-effective method which helps maintain disinfectant levels throughout the system [26]. Although the periods of contamination decrease were long, this method could represent a valid choice for improving drinking water quality and disinfectant efficacy, reducing the critical section of water plants and biofilm maturation in dead-end branches. 

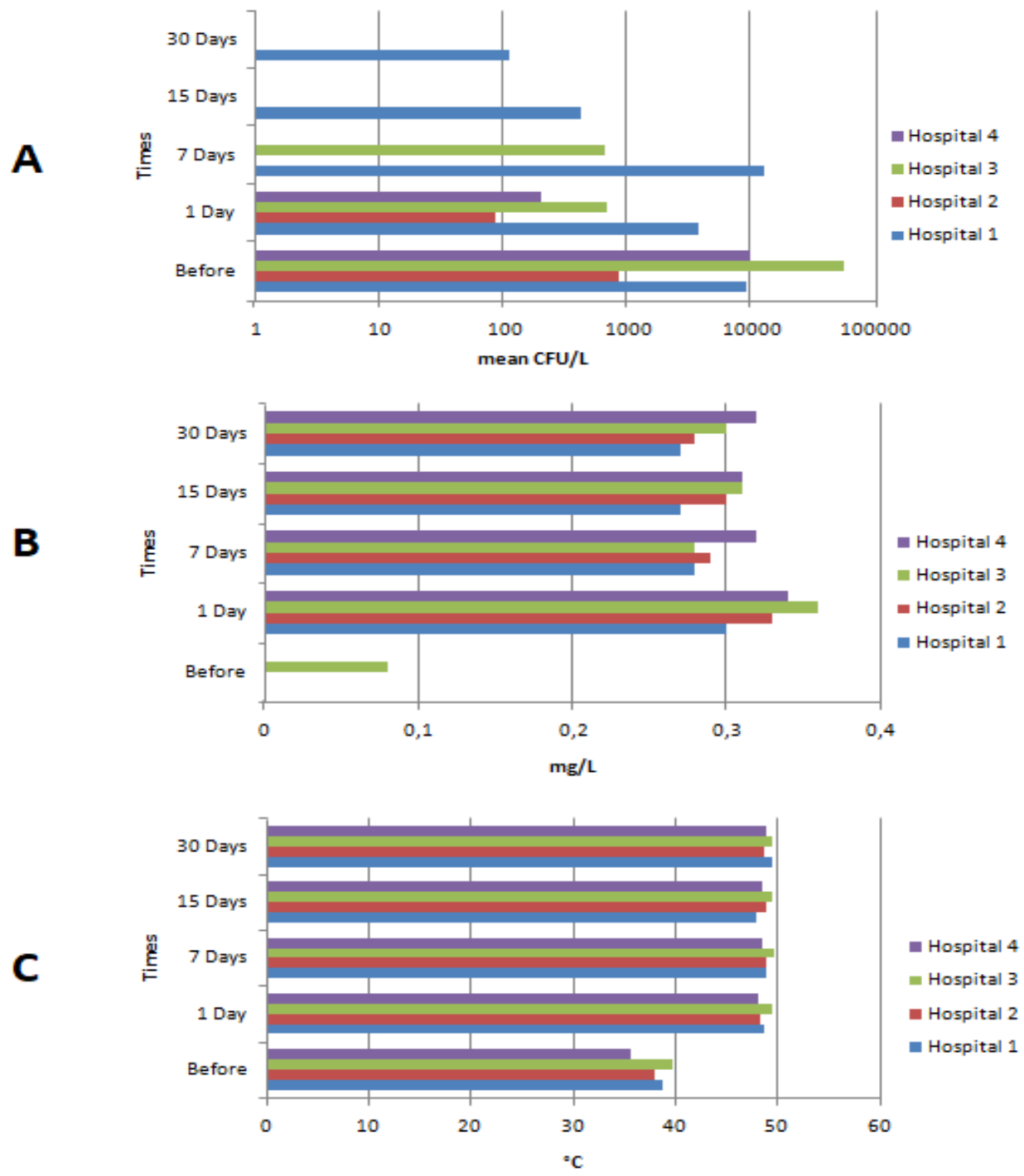

Figure 5. Legionella pneumophila counts (CFU/L) (A), total chlorine concentration (mg/L) (B), and temperature values $\left({ }^{\circ} \mathrm{C}\right)(\mathrm{C})$ detected in all the hospitals.

Author Contributions: A.B. and G.P. conceived and designed the experiments. M.T., B.C., P.V. and A.L.C. performed the experiments and wrote the paper. T.M., C.B. and E.D.V. performed the water samplings. All authors have read and agreed to the published version of the manuscript.

Funding: This research received no external funding.

Conflicts of Interest: All authors have no conflict of interest to declare.

\section{References}

1. Oda, N.; Hirahara, T.; Fujioka, Y.; Mitani, R.; Takata, I. Legionella Pneumonia Following the Heavy Rain Event of July 2018 in Japan. Intern Med. 2019, 58, 2831-2834. [CrossRef] [PubMed]

2. Muldrow, L.L.; Tyndall, R.L.; Fliermans, C.B. Application of flow cytometry to studies of pathogenic free-living amoebae. Appl. Environ. Microbiol. 1982, 44, 1258-1269. [CrossRef] [PubMed]

3. European Centre for Disease Prevention and Control. Legionnaires' Disease. Annual Epidemiological Report for 2017; European Centre for Disease Prevention and Control Stockholm: Solna, Sweden, 2019; pp. 1-6. Available online: https://www.ecdc.europa.eu/en/publications-data/legionnaires-disease-annual-epidemiologicalreport-2017 (accessed on 12 November 2019). 
4. Alexandropoulou, I.G.; Ntougias, S.; Konstantinidis, T.G.; Parasidis, T.A.; Panopoulou, M.; Constantinidis, T.C. Environmental surveillance and molecular epidemiology of waterborne pathogen Legionella pneumophila in health-care facilities of Northeastern Greece: A 4-year survey. Environ. Sci. Pollut. Res. Int. 2015, 22, 7628-7640. [CrossRef] [PubMed]

5. Superior Institute of Health. Rapporto Annuale Sulla Legionellosi in Italia Nel 2017; Superior Institute of Health: Rome, Italy, 2018; Volume 31, pp. 1-24. Available online: http://www.legionellaonline.it/notiziari_ISS.htm (accessed on 12 November 2019).

6. World Health Organization. Legionella and the Prevention of Legionellosis; World Health Organization: Geneva, Switzerland, 2007; pp. 1-276. Available online: https:/www.who.int/water_sanitation_health/publications/ legionella/en/ (accessed on 12 November 2019).

7. Superior Institute of Health. Linee guida per la prevenzione ed il controllo della legionellosi; Superior Institute of Health: Rome, Italy, 2015; pp. 1-149. Available online: http://www.salute.gov.it/portale/documentazione/p6_ 2_2_1.jsp?id=2362 (accessed on 12 November 2019).

8. Whiley, H.; Hinds, J.; Xi, J.; Bentham, R. Real-Time Continuous Surveillance of Temperature and Flow Events Presents a Novel Monitoring Approach for Hospital and Healthcare Water Distribution Systems. Int. J. Environ. Res. Public Health 2019, 16, 1332. [CrossRef] [PubMed]

9. Rhoads, W.J.; Pruden, A.; Edwards, M.A. Interactive Effects of Corrosion, Copper, and Chloramines on Legionella and Mycobacteria in Hot Water Plumbing. Environ. Sci. Technol. 2017, 51, 7065-7075. [CrossRef] [PubMed]

10. Flemming, H.C.; Wingender, J. The biofilm matrix. Nat. Rev. Microbiol. 2010, 8, 623-633. [CrossRef] [PubMed]

11. Manuel, C.M.; Nunes, O.C.; Melo, L.F. Dynamics of drinking water biofilm in flow/non-flow conditions. Water Res. 2007, 41, 551-562. [CrossRef] [PubMed]

12. Totaro, M.; Valentini, P.; Costa, A.L.; Giorgi, S.; Casini, B.; Baggiani, A. Rate of Legionella pneumophila colonization in hospital hot water network after time flow taps installation. J. Hosp. Infect. 2018, 98, 60-63. [CrossRef] [PubMed]

13. International Organization for Standardization. ISO 11731 Water quality-Enumeration of Legionella; International Organization for Standardization: Geneva, Switzerland, 2017; pp. 1-38. Available online: https://www.iso.org/standard/61782.html (accessed on 19 November 2019).

14. The Council of the European Union. Council Directive 98/83/EC of 3 November 1998 on the Quality of Water Intended for Human Consumption; The Council of the European Union: Bruxelles, Belgium, 1998; pp. 1-23. Available online: https://op.europa.eu/en/publication-detail/-/publication/880a8575-7ee4-438e-b953-567df6719e26 (accessed on 19 November 2019).

15. Montagna, M.T.; De Giglio, O.; Cristina, M.L.; Napoli, C.; Pacifico, C.; Agodi, A.; Baldovin, T.; Casini, B.; Coniglio, M.A.; D'Errico, M.M.; et al. Evaluation of Legionella Air Contamination in Healthcare Facilities by Different Sampling Methods: An Italian Multicenter Study. Int. J. Environ. Res. Public Health 2017, 14, 670. [CrossRef] [PubMed]

16. Kanamori, H.; Weber, D.J.; Rutala, W.A. Healthcare Outbreaks Associated with a Water Reservoir and Infection Prevention Strategies. Clin. Infect. Dis. 2016, 62, 1423-1435. [CrossRef] [PubMed]

17. Totaro, M.; Casini, B.; Valentini, P.; Miccoli, M.; Giorgi, S.; Porretta, A.; Privitera, G.; Lopalco, P.L.; Baggiani, A. Evaluation and control of microbial and chemical contamination in dialysis water plants of Italian nephrology wards. J. Hosp. Infect. 2017, 97, 169-174. [CrossRef] [PubMed]

18. Scaturro, M.; Dell'eva, I.; Helfer, F.; Ricci, M.L. Persistence of the same strain of Legionella pneumophila in the water system of an Italian hospital for 15 years. Infect. Control Hosp. Epidemiol. 2007, 28, 1089-1092. [CrossRef] [PubMed]

19. Grayman, W.M. A quarter of a century of water quality modeling in distribution systems. In Eighth Annual Water Distribution Systems Analysis Symposium (WDSA); American Society of Civil Engineers: Cincinnati, OH, USA, 2012; Available online: https://ascelibrary.org/doi/abs/10.1061/40941\%28247\%294 (accessed on 19 November 2019).

20. Gavaldà, L.; Garcia-Nuñez, M.; Quero, S.; Gutierrez-Milla, C.; Sabrià, M. Role of hot water temperature and water system use on Legionella control in a tertiary hospital: An 8-year longitudinal study. Water Res. 2019, 149, 460-466. [CrossRef] [PubMed]

21. Bédard, E.; Laferrière, C.; Déziel, E.; Prévost, M. Impact of stagnation and sampling volume on water microbial quality monitoring in large buildings. PLOS ONE 2018, 13, e0199429. [CrossRef] [PubMed] 
22. Fish, K.; Osborn, A.M.; Boxall, J.B. Biofilm structures (EPS and bacterial communities) in drinking water distribution systems are conditioned by hydraulics and influence discolouration. Sci. Total Environ. 2017, 593, 571-580. [CrossRef] [PubMed]

23. Totaro, M.; Valentini, P.; Casini, B.; Miccoli, M.; Costa, A.L.; Baggiani, A. Experimental comparison of point-of-use filters for drinking water ultrafiltration. J. Hosp. Infect. 2017, 96, 172-176. [CrossRef] [PubMed]

24. Szabo, J.; Minamyer, S. Decontamination of biological agents from drinking water infrastructure: A literature review and summary. Environ. Int. 2014, 72, 124-128. [CrossRef] [PubMed]

25. Manuel, C.M.; Nunes, O.C.; Melo, L.F. Unsteady state flow and stagnation in distribution systems affect the biological stability of drinking water. Biofouling 2010, 26, 129-139. [CrossRef] [PubMed]

26. Totaro, M.; De Vita, E.; Mariotti, T.; Bisordi, C.; Giorgi, S.; Gallo, A.; Costa, A.L.; Casini, B.; Valentini, P.; Privitera, G.; et al. Cost analysis for electron time-flow taps and point of use filters: A comparison of two methods for Legionnaires' disease prevention in hospital water networks. J. Hosp. Infect. 2019, 103, 231-232. [CrossRef] [PubMed]

(C) 2020 by the authors. Licensee MDPI, Basel, Switzerland. This article is an open access article distributed under the terms and conditions of the Creative Commons Attribution (CC BY) license (http://creativecommons.org/licenses/by/4.0/). 